\title{
A Diffusion Process with a Brownian Potential Including a Zero Potential Part
}

\author{
Yuki SUZUKI
}

Keio University

\begin{abstract}
A one-dimensional diffusion process with a Brownian potential including a zero potential part is studied. The maximum process and the minimum process of the diffusion process are also investigated.
\end{abstract}

\section{Model and results}

Denote by $\mathbb{W}$ the space of real-valued continuous functions on $\mathbb{R}$ vanishing at 0 , and $\widetilde{\mathbb{W}}$ the space of real-valued right-continuous functions on $\mathbb{R}$ with left limits. Let $a \in(0,1 / 2)$ be fixed. For $w \in \mathbb{W}$ and $\lambda>0$, define $T_{\lambda} w \in \widetilde{\mathbb{W}}$ by

$$
\left(T_{\lambda} w\right)(x)= \begin{cases}0 & \text { for } 0<x<e^{a \lambda}, \\ w(x) & \text { otherwise. }\end{cases}
$$

We denote by $\Omega$ the space of real-valued continuous functions on $[0, \infty)$, and for $\omega \in \Omega$ and $t \geq 0$ we write $X(t)=X(t, \omega)=\omega(t)$, the value of $\omega$ at $t$. For $w \in \mathbb{W}, \lambda>0$ and $x_{0} \in \mathbb{R}, P_{T_{\lambda} w}^{x_{0}}$ denotes the probability measure on $\Omega$ such that $\left\{X(t), t \geq 0, P_{T_{\lambda} w}^{x_{0}}\right\}$ is a diffusion process with generator

$$
\mathcal{L}_{T_{\lambda} w}=\frac{1}{2} e^{\left(T_{\lambda} w\right)(x)} \frac{d}{d x}\left(e^{-\left(T_{\lambda} w\right)(x)} \frac{d}{d x}\right)
$$

starting from $x_{0}$. Let $P$ be the Wiener measure on $\mathbb{W}$, and $\mathcal{P}_{\lambda}^{x_{0}}$ be the probability measure on $\mathbb{W} \times \Omega$ defined by

$$
\mathcal{P}_{\lambda}^{x_{0}}(d w d \omega)=P(d w) P_{T_{\lambda} w}^{x_{0}}(d \omega) .
$$

For each $\lambda>0$ we regard the process $\left\{X(t), t \geq 0, \mathcal{P}_{\lambda}^{x_{0}}\right\}$ as one defined on the probability space $\left(\mathbb{W} \times \Omega, \mathcal{P}_{\lambda}^{x_{0}}\right)$, which we call a diffusion process with a Brownian potential including a zero potential part. We study the behavior of the process $\left\{X(t), t \geq 0, \mathcal{P}_{\lambda}^{0}\right\}$ at $t=e^{\lambda}$ $(\lambda \rightarrow \infty)$.

Received December 26, 2013; revised August 6, 2014 2010 Mathematics Subject Classification: 60J60, 60K37

Key words and phrases: Random environment, Diffusion process 
Brox ([1]) and Schumacher ([7]) studied a one-dimensional diffusion process with a Brownian potential, and Kawazu, Tamura and Tanaka ([5], [6]) investigated a onedimensional diffusion process in an asymptotically self-similar random environment. Moreover, in [4] and [3] a one-dimensional diffusion process with a one-sided Brownian potential was studied, and in [8] a one-dimensional diffusion process with a random potential consisting of two self-similar processes with different indices for the right and the left hand sides of the origin was investigated.

Our present model is a variant of the diffusion in [1], [7] and also that in [4], [3]. To study the behavior of our process $\left\{X(t), t \geq 0, \mathcal{P}_{\lambda}^{0}\right\}$ at $t=e^{\lambda}$, we regard $\mathbb{W}$ as a disjoint union of three subsets $\mathbb{A}_{\lambda}, \mathbb{B}_{\lambda}$ and $\mathbb{C}_{\lambda}$; for the definition, see (1.3)-(1.5). We show that $X\left(e^{\lambda}\right)$ exhibits quite different behavior depending on whether it is conditioned on $\mathbb{A}_{\lambda}, \mathbb{B}_{\lambda}$ or $\mathbb{C}_{\lambda}$ $(\lambda \rightarrow \infty)$. The behavior of $X\left(e^{\lambda}\right)$ conditioned on $\mathbb{B}_{\lambda}$ is much different from the result in [1], [7]. Roughly speaking, in this case with high probability $X\left(e^{\lambda}\right)$ is not at the bottom of the "valley" but in the interval where the potential is identically zero; for the precise meaning of this, see Theorem 1.1 (ii).

Hereafter we restrict $\mathbb{W}$ to a suitable subset of $\mathbb{W}$ that has $P$-measure 1 to avoid unpleasant cases. For $w \in \mathbb{W}$ and $\rho \in \mathbb{R}$, we set

$$
\begin{aligned}
& \sigma(\rho)=\sigma(\rho, w)=\sup \{x<0: w(x)=\rho\}, \\
& \zeta=\zeta(w)=\sup \left\{x<0: w(x)-\min _{x \leq y \leq 0} w(y)=1\right\}, \\
& V=V(w)=\min _{\zeta \leq x \leq 0} w(x) .
\end{aligned}
$$

We define $b=b(w) \in(\zeta, 0)$ by $w(b)=V$. We note that $b$ is determined uniquely by $w$ (P-a.s.).

To study the behavior of our process, we regard $\mathbb{W}$ as a disjoint union of three subsets $\mathbb{A}, \mathbb{B}$ and $\mathbb{C}$ defined by

$$
\begin{aligned}
& \mathbb{A}=\mathbb{A}^{\prime} \cup \mathbb{A}^{\prime \prime}, \\
& \mathbb{A}^{\prime}=\{w \in \mathbb{W}: \sigma(a)<\sigma(-1+a)\}, \\
& \mathbb{A}^{\prime \prime}=\{w \in \mathbb{W}: w(1)>0, \sigma(1-a)<\sigma(-a)\}, \\
& \mathbb{B}=\{w \in \mathbb{W}: w(1)>0, \sigma(-a)<\sigma(1-a)\}, \\
& \mathbb{C}=\{w \in \mathbb{W}: w(1)<0, \sigma(-1+a)<\sigma(a)\} .
\end{aligned}
$$

(See Fig. 1-Fig. 4 in the next page.) We note $\mathbb{A}^{\prime} \cap \mathbb{A}^{\prime \prime}=\mathbb{A}^{\prime} \cap\{w \in \mathbb{W}: w(1)>0\}$ and have $P\left\{\mathbb{A}^{\prime}\right\}=a, P\left\{\mathbb{A}^{\prime \prime}\right\}=(1-a) / 2, P\{\mathbb{A}\}=1 / 2, P\{\mathbb{B}\}=a / 2$ and $P\{\mathbb{C}\}=(1-a) / 2$. Moreover, we remark that

$$
V(w)<-a \text { if } w \in \mathbb{A} .
$$




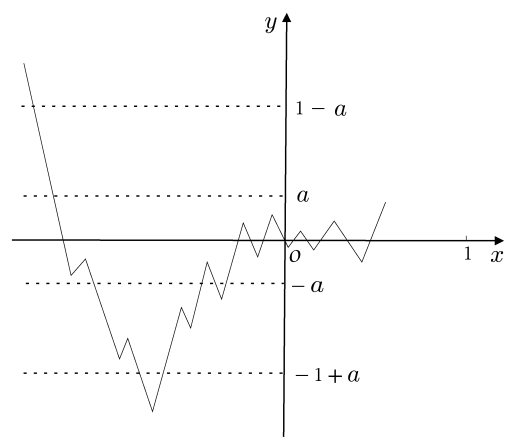

Fig. 1. $w \in \mathbb{A}^{\prime}$

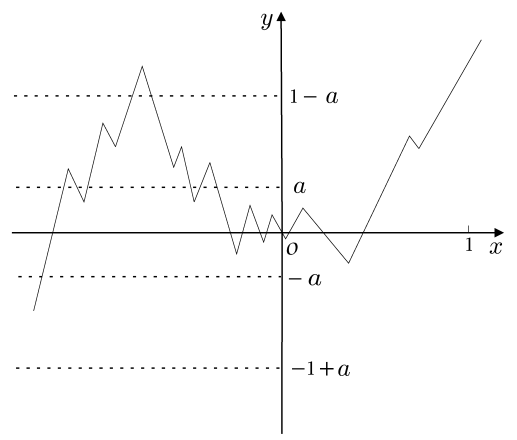

Fig. 3. $w \in \mathbb{B}$

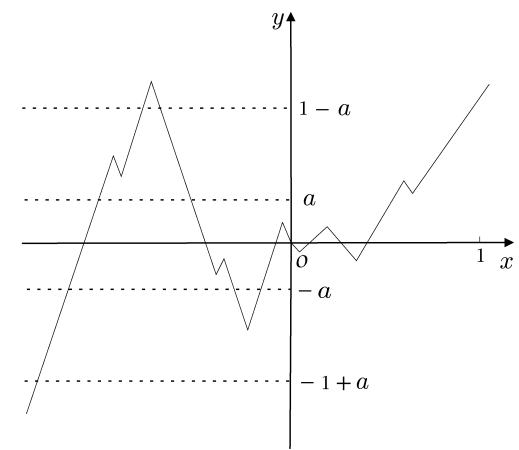

Fig. 2. $w \in \mathbb{A}^{\prime \prime} \cap\left(\mathbb{A}^{\prime}\right)^{c}$

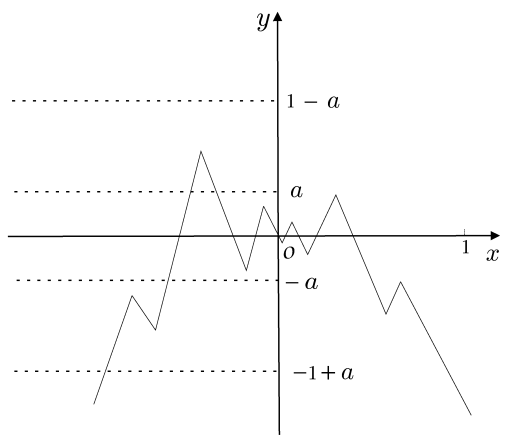

Fig. 4. $w \in \mathbb{C}$

For $w \in \mathbb{W}$ and $\lambda>0$, we define $\tau_{\lambda} w \in \mathbb{W}$ by

$$
\left(\tau_{\lambda} w\right)(x)= \begin{cases}\lambda^{-1} w\left(\lambda^{2} x\right) & \text { for } x \leq 0, \\ e^{-a \lambda / 2} w\left(e^{a \lambda} x\right) & \text { for } x>0 .\end{cases}
$$

Note that

$$
\left\{\tau_{\lambda} w, P\right\} \stackrel{\mathrm{d}}{=}\{w, P\}
$$

where $\stackrel{\mathrm{d}}{=}$ means the equality in distribution. To state our result, for each $\lambda>0$ we regard $\mathbb{W}$ as a disjoint union of three subsets $\mathbb{A}_{\lambda}, \mathbb{B}_{\lambda}$ and $\mathbb{C}_{\lambda}$ defined by

$$
\begin{aligned}
& \mathbb{A}_{\lambda}=\left\{w \in \mathbb{W}: \tau_{\lambda} w \in \mathbb{A}\right\}, \\
& \mathbb{B}_{\lambda}=\left\{w \in \mathbb{W}: \tau_{\lambda} w \in \mathbb{B}\right\}, \\
& \mathbb{C}_{\lambda}=\left\{w \in \mathbb{W}: \tau_{\lambda} w \in \mathbb{C}\right\} .
\end{aligned}
$$


(See [3], [8].) We notice that each $P$-measure of $\mathbb{A}_{\lambda}, \mathbb{B}_{\lambda}$ and $\mathbb{C}_{\lambda}$ is equal to that of $\mathbb{A}, \mathbb{B}$ and $\mathbb{C}$, respectively.

In the following theorems, we denote by $P\{\cdots \mid \cdot\}$ the conditional probability.

THEOREM 1.1. For any $\varepsilon>0$ the following (i)-(iii) hold.

(i) $\lim _{\lambda \rightarrow \infty} P\left\{\mathbb{E}_{1, \lambda, \varepsilon} \mid \mathbb{A}_{\lambda}\right\}=1$,

where

$$
\begin{aligned}
& \mathbb{E}_{1, \lambda, \varepsilon}=\left\{w \in \mathbb{W}: p_{1, \lambda, \varepsilon}(w)>1-\varepsilon\right\}, \\
& p_{1, \lambda, \varepsilon}(w)=P_{T_{\lambda} w}^{0}\left\{b\left(\tau_{\lambda} w\right)-\varepsilon<\lambda^{-2} X\left(e^{\lambda}\right)<\left(b\left(\tau_{\lambda} w\right)+\varepsilon\right) \wedge 0\right\} .
\end{aligned}
$$

(ii) $\lim _{\lambda \rightarrow \infty} P\left\{\mathbb{E}_{2, \lambda, \varepsilon} \mid \mathbb{B}_{\lambda}\right\}=1$,

where

$$
\begin{aligned}
& \mathbb{E}_{2, \lambda, \varepsilon}=\left\{w \in \mathbb{W}: p_{2, \lambda}(w)>1-\varepsilon\right\}, \\
& p_{2, \lambda}(w)=P_{T_{\lambda} w}^{0}\left\{0<e^{-a \lambda} X\left(e^{\lambda}\right)<1\right\} .
\end{aligned}
$$

(iii) $\lim _{\lambda \rightarrow \infty} P\left\{\mathbb{E}_{3, \lambda, \varepsilon} \mid \mathbb{C}_{\lambda}\right\}=1$,

where

$$
\begin{aligned}
& \mathbb{E}_{3, \lambda, \varepsilon}=\left\{w \in \mathbb{W}: p_{3, \lambda, \varepsilon}(w)>1-\varepsilon\right\}, \\
& p_{3, \lambda, \varepsilon}(w)=P_{T_{\lambda} w}^{0}\left\{-\left(\exp \left\{w\left(e^{a \lambda}\right)+e^{a \lambda / 2} \varepsilon\right\} \wedge \varepsilon\right)<e^{-a \lambda} X\left(e^{\lambda}\right)-1<\varepsilon\right\} .
\end{aligned}
$$

The following corollary concerning the occupation time is obtained from the proof of Theorem 1.1 (cf. [3]). In the following, $\mathbf{1}_{E}$ denotes the indicator function of the (generic) set E.

COROLlary 1.2. For any $\varepsilon>0$ the following (i)-(iii) hold.

(i) $\lim _{\lambda \rightarrow \infty} P\left\{\mathbb{E}_{4, \lambda, \varepsilon} \mid \mathbb{A}_{\lambda}\right\}=1$,

where

$$
\begin{aligned}
& \mathbb{E}_{4, \lambda, \varepsilon}=\left\{w \in \mathbb{W}: p_{4, \lambda, \varepsilon}(w)>1-\varepsilon\right\}, \\
& p_{4, \lambda, \varepsilon}(w)=P_{T_{\lambda} w}^{0}\left\{e^{-\lambda} \int_{0}^{e^{\lambda}} \mathbf{1}_{\left(b\left(\tau_{\lambda} w\right)-\varepsilon,\left(b\left(\tau_{\lambda} w\right)+\varepsilon\right) \wedge 0\right)}\left(\lambda^{-2} X(t)\right) d t>1-\varepsilon\right\} .
\end{aligned}
$$

(ii) $\lim _{\lambda \rightarrow \infty} P\left\{\mathbb{E}_{5, \lambda, \varepsilon} \mid \mathbb{B}_{\lambda}\right\}=1$,

where

$$
\begin{aligned}
& \mathbb{E}_{5, \lambda, \varepsilon}=\left\{w \in \mathbb{W}: p_{5, \lambda, \varepsilon}(w)>1-\varepsilon\right\} \\
& p_{5, \lambda, \varepsilon}(w)=P_{T_{\lambda} w}^{0}\left\{e^{-\lambda} \int_{0}^{e^{\lambda}} \mathbf{1}_{(0,1)}\left(e^{-a \lambda} X(t)\right) d t>1-\varepsilon\right\} .
\end{aligned}
$$

(iii) $\lim _{\lambda \rightarrow \infty} P\left\{\mathbb{E}_{6, \lambda, \varepsilon} \mid \mathbb{C}_{\lambda}\right\}=1$, 
where

$$
\begin{aligned}
& \mathbb{E}_{6, \lambda, \varepsilon}=\left\{w \in \mathbb{W}: p_{6, \lambda, \varepsilon}(w)>1-\varepsilon\right\}, \\
& p_{6, \lambda, \varepsilon}(w)=P_{T_{\lambda} w}^{0}\left\{e^{-\lambda} \int_{0}^{e^{\lambda}} \mathbf{1}_{\left(1-\exp \left\{w\left(e^{a \lambda}\right)+e^{a \lambda / 2} \varepsilon\right\} \wedge \varepsilon, 1+\varepsilon\right)}\left(e^{-a \lambda} X(t)\right) d t>1-\varepsilon\right\} .
\end{aligned}
$$

Next we consider the maximum process and the minimum process of $\left\{X(t), t \geq 0, \mathcal{P}_{\lambda}^{0}\right\}$. For $\omega \in \Omega$, we set $\bar{X}(t)=\bar{X}(t, \omega)=\max _{0 \leq s \leq t} X(s, \omega)$ and $\underline{X}(t)=\underline{X}(t, \omega)=$ $\min _{0 \leq s \leq t} X(s, \omega)$. We study the behaviors of the processes $\left\{\bar{X}(t), t \geq 0, \mathcal{P}_{\lambda}^{0}\right\}$ and $\{\underline{X}(t), t \geq$ $\left.0, \mathcal{P}_{\lambda}^{0}\right\}$ at $t=e^{\lambda}$.

To study the behavior of the maximum process, we set, for $w \in \mathbb{W}$,

$$
\begin{aligned}
& H=H(w)=\max _{\zeta \leq x \leq 0} w(x), \\
& M=M(w)=\max _{b \leq x \leq 0} w(x) .
\end{aligned}
$$

Note that

$$
H(w)<a \text { if } w \in \mathbb{A}^{\prime} .
$$

We divide $\mathbb{A}^{\prime}$ into two subsets $\mathbb{A}_{I}^{\prime}$ and $\mathbb{A}_{I I}^{\prime}$ as follows:

$$
\begin{aligned}
& \mathbb{A}_{I}^{\prime}=\left\{w \in \mathbb{A}^{\prime}: M \leq V+1\right\}, \\
& \mathbb{A}_{I I}^{\prime}=\left\{w \in \mathbb{A}^{\prime}: M>V+1\right\} .
\end{aligned}
$$

(See Fig. 5 and Fig. 6 below.) Moreover, we set

$$
\mathbb{D}=\left(\mathbb{A}^{\prime \prime} \cap\left(\mathbb{A}^{\prime}\right)^{c}\right) \oplus \mathbb{B} .
$$

(See Fig. 2 and Fig. 3.) We have $P\{\mathbb{D}\}=(1-a) / 2$.

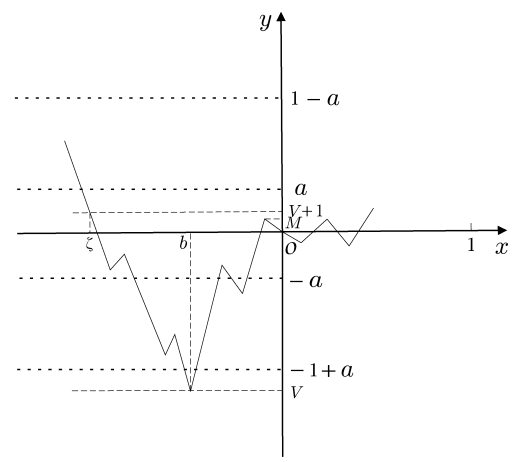

Fig. 5. $w \in \mathbb{A}_{I}^{\prime}$

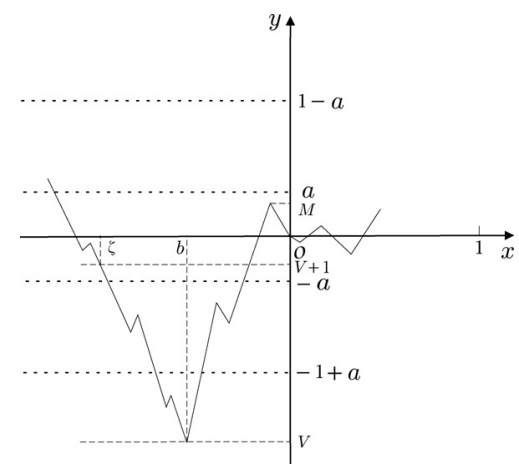

Fig. 6. $\quad w \in \mathbb{A}_{I I}^{\prime}$ 
To state our result on the maximum process, for each $\lambda>0$ we regard $\mathbb{W}$ as a disjoint union of four subsets $\mathbb{A}_{I, \lambda}^{\prime}, \mathbb{A}_{I I, \lambda}^{\prime}, \mathbb{D}_{\lambda}$ and $\mathbb{C}_{\lambda}$, where

$$
\begin{aligned}
& \mathbb{A}_{I, \lambda}^{\prime}=\left\{w \in \mathbb{W}: \tau_{\lambda} w \in \mathbb{A}_{I}^{\prime}\right\}, \\
& \mathbb{A}_{I I, \lambda}^{\prime}=\left\{w \in \mathbb{W}: \tau_{\lambda} w \in \mathbb{A}_{I I}^{\prime}\right\}, \\
& \mathbb{D}_{\lambda}=\left\{w \in \mathbb{W}: \tau_{\lambda} w \in \mathbb{D}\right\} .
\end{aligned}
$$

Note that each $P$-measure of $\mathbb{A}_{I, \lambda}^{\prime}, \mathbb{A}_{I I, \lambda}^{\prime}$ and $\mathbb{D}_{\lambda}$ is equal to that of $\mathbb{A}_{I}^{\prime}, \mathbb{A}_{I I}^{\prime}$ and $\mathbb{D}$, respectively.

THEOREM 1.3. For any $\varepsilon>0$ the following (i)-(iv) hold.

(i) $\lim _{\lambda \rightarrow \infty} P\left\{\mathbb{E}_{7, \lambda, \varepsilon} \mid \mathbb{A}_{I, \lambda}^{\prime}\right\}=1$,

where

$$
\begin{aligned}
& \mathbb{E}_{7, \lambda, \varepsilon}=\left\{w \in \mathbb{W}: p_{7, \lambda, \varepsilon}(w)>1-\varepsilon\right\}, \\
& p_{7, \lambda, \varepsilon}(w)=P_{T_{\lambda} w}^{0}\left\{e^{\lambda\left(H\left(\tau_{\lambda} w\right)-\varepsilon\right)}<\bar{X}\left(e^{\lambda}\right)<e^{\lambda\left(H\left(\tau_{\lambda} w\right)+\varepsilon\right)} \wedge e^{a \lambda} \varepsilon\right\} .
\end{aligned}
$$

(ii) $\lim _{\lambda \rightarrow \infty} P\left\{\mathbb{E}_{8, \lambda, \varepsilon} \mid \mathbb{A}_{I I, \lambda}^{\prime}\right\}=1$,

where

$$
\begin{aligned}
& \mathbb{E}_{8, \lambda, \varepsilon}=\left\{w \in \mathbb{W}: p_{8, \lambda, \varepsilon}(w)>1-\varepsilon\right\}, \\
& p_{8, \lambda, \varepsilon}(w)=P_{T_{\lambda} w}^{0}\left\{e^{\lambda\left(H\left(\tau_{\lambda} w\right)-\varepsilon\right)}<\bar{X}\left(e^{\lambda}\right)<e^{\lambda\left(H\left(\tau_{\lambda} w\right)+\varepsilon(\lambda)\right)} \wedge e^{a \lambda} \varepsilon\right\},
\end{aligned}
$$

and $\varepsilon(\lambda)>0, \lambda>0$, is assumed to satisfy $\lim _{\lambda \rightarrow \infty} \varepsilon(\lambda)=0$ and $\liminf _{\lambda \rightarrow \infty} \lambda(\log \lambda)^{-1} \varepsilon(\lambda)>2$.

$$
\text { (iii) } \lim _{\lambda \rightarrow \infty} P\left\{\mathbb{E}_{9, \lambda, \varepsilon} \mid \mathbb{D}_{\lambda}\right\}=1 \text {, }
$$

where

$$
\begin{aligned}
& \mathbb{E}_{9, \lambda, \varepsilon}=\left\{w \in \mathbb{W}: p_{9, \lambda, \varepsilon}(w)>1-\varepsilon\right\}, \\
& p_{9, \lambda, \varepsilon}(w) \\
& =P_{T_{\lambda} w}^{0}\left\{\exp \left\{-w\left(e^{a \lambda}\right)-e^{a \lambda / 2} \varepsilon\right\}<e^{-a \lambda} \bar{X}\left(e^{\lambda}\right)-1<\exp \left\{-w\left(e^{a \lambda}\right)+e^{a \lambda / 2} \varepsilon\right\} \wedge \varepsilon\right\} .
\end{aligned}
$$

$$
\text { (iv) } \lim _{\lambda \rightarrow \infty} P\left\{\mathbb{E}_{10, \lambda, \varepsilon} \mid \mathbb{C}_{\lambda}\right\}=1 \text {, }
$$

where

$$
\begin{aligned}
& \mathbb{E}_{10, \lambda, \varepsilon}=\left\{w \in \mathbb{W}: p_{10, \lambda, \varepsilon}(w)>1-\varepsilon\right\}, \\
& p_{10, \lambda, \varepsilon}(w)=P_{T_{\lambda} w}^{0}\left\{\exp \left\{-e^{a \lambda / 2} \varepsilon\right\}<e^{-a \lambda} \bar{X}\left(e^{\lambda}\right)-1<\varepsilon\right\} .
\end{aligned}
$$

To study the behavior of the minimum process, we set, for $w \in \mathbb{W}$ and $\gamma>0$,

$$
\zeta_{\gamma}=\zeta_{\gamma}(w)=\sup \left\{x<b(w): w(x)-\min _{x \leq y \leq 0} w(y)=\gamma\right\} .
$$


Notice that $\zeta_{1}=\zeta$. The following theorem is concerning the minimum process, where we have more precise upper bound of $\underline{X}\left(e^{\lambda}\right)$ than the corresponding result in [4].

THEOREM 1.4. Let $\varepsilon>0$ and $\varepsilon(\lambda)>0, \lambda>0$, satisfy $\lim _{\lambda \rightarrow \infty} \varepsilon(\lambda)=0$.

(i) $\lim _{\lambda \rightarrow \infty} P\left\{\mathbb{E}_{11, \lambda, \varepsilon} \mid \mathbb{A}_{\lambda}\right\}=1$,

where

$$
\begin{aligned}
& \mathbb{E}_{11, \lambda, \varepsilon}=\left\{w \in \mathbb{W}: p_{11, \lambda, \varepsilon}(w)>1-\varepsilon\right\}, \\
& p_{11, \lambda, \varepsilon}(w)=P_{T_{\lambda} w}^{0}\left\{\zeta_{1+\varepsilon}\left(\tau_{\lambda} w\right)<\lambda^{-2} \underline{X}\left(e^{\lambda}\right)<\zeta_{1-\varepsilon(\lambda)}\left(\tau_{\lambda} w\right)\right\},
\end{aligned}
$$

and $\varepsilon(\lambda), \lambda>0$, is assumed additionally to satisfy $\liminf _{\lambda \rightarrow \infty} \lambda(\log \lambda)^{-1} \varepsilon(\lambda)>4$.

(ii) $\lim _{\lambda \rightarrow \infty} P\left\{\mathbb{E}_{12, \lambda, \varepsilon} \mid \mathbb{B}_{\lambda}\right\}=1$,

where

$$
\begin{aligned}
& \mathbb{E}_{12, \lambda, \varepsilon}=\left\{w \in \mathbb{W}: p_{12, \lambda, \varepsilon}(w)>1-\varepsilon\right\}, \\
& p_{12, \lambda, \varepsilon}(w)=P_{T_{\lambda} w}^{0}\left\{\sigma\left(1-a+\varepsilon, \tau_{\lambda} w\right)<\lambda^{-2} \underline{X}\left(e^{\lambda}\right)<\sigma\left(1-a-\varepsilon(\lambda), \tau_{\lambda} w\right)\right\},
\end{aligned}
$$

and $\varepsilon(\lambda), \lambda>0$, is assumed additionally to satisfy $\liminf _{\lambda \rightarrow \infty} \lambda(\log \lambda)^{-1} \varepsilon(\lambda)>2$.

(iii) $\lim _{\lambda \rightarrow \infty} P\left\{\mathbb{E}_{13, \lambda, \varepsilon} \mid \mathbb{C}_{\lambda}\right\}=1$,

where

$$
\begin{aligned}
& \mathbb{E}_{13, \lambda, \varepsilon}=\left\{w \in \mathbb{W}: p_{13, \lambda, \varepsilon}(w)>1-\varepsilon\right\}, \\
& p_{13, \lambda, \varepsilon}(w)=P_{T_{\lambda} w}^{0}\left\{\sigma\left(a, \tau_{\lambda} w\right)<\lambda^{-2} \underline{X}\left(e^{\lambda}\right)<\sigma\left(a-\varepsilon(\lambda), \tau_{\lambda} w\right)\right\},
\end{aligned}
$$

and $\varepsilon(\lambda), \lambda>0$, is assumed additionally to satisfy $\liminf _{\lambda \rightarrow \infty} \lambda(\log \lambda)^{-1} \varepsilon(\lambda)>2$.

\section{Preliminaries}

For $w \in \mathbb{W}$ and $\lambda>0$, define $G_{\lambda} w \in \widetilde{\mathbb{W}}$ by

$$
\left(G_{\lambda} w\right)(x)= \begin{cases}\lambda w\left(\lambda^{-2} e^{a \lambda} x\right) & \text { for } x \leq 0, \\ 0 & \text { for } 0<x<1, \\ e^{a \lambda / 2} w(x) & \text { for } x \geq 1\end{cases}
$$

For $x_{0} \in \mathbb{R}, P_{G_{\lambda} w}^{x_{0}}$ denotes the probability measure on $\Omega$ such that $\left\{X(t), t \geq 0, P_{G_{\lambda} w}^{x_{0}}\right\}$ is a diffusion process with generator

$$
\mathcal{L}_{G_{\lambda} w}=\frac{1}{2} e^{\left(G_{\lambda} w\right)(x)} \frac{d}{d x}\left(e^{-\left(G_{\lambda} w\right)(x)} \frac{d}{d x}\right)
$$

starting from $x_{0}$. We can construct such a diffusion process on a probability space $(\widetilde{\Omega}, \widetilde{P})$ as follows ([2], see also [4], [8]). Let $\{B(t), t \geq 0\}$ be a one-dimensional Brownian motion 
starting from 0 defined on $(\widetilde{\Omega}, \widetilde{P})$, and set

$$
L(t, x)=\lim _{\varepsilon \downarrow 0} \frac{1}{\varepsilon} \int_{0}^{t} \mathbf{1}_{[x, x+\varepsilon)}(B(s)) d s, \quad t \geq 0, x \in \mathbb{R} \quad \text { (local time) } .
$$

We also set

$$
\begin{aligned}
& S_{G_{\lambda} w}(x)=\int_{0}^{x} e^{\left(G_{\lambda} w\right)(y)} d y, \quad x \in \mathbb{R}, \\
& \begin{aligned}
& A_{G_{\lambda} w}(t)=\int_{0}^{t} e^{-2\left(G_{\lambda} w\right)\left(S_{\left.G_{\lambda} w^{-1}(B(s))\right)}\right.} d s \\
&=\int_{-\infty}^{\infty} e^{-2\left(G_{\lambda} w\right)\left(S_{\left.G_{\lambda} w^{-1}(x)\right)} L(t, x) d x, \quad t \geq 0,\right.} \\
& X\left(t ; 0, G_{\lambda} w\right)=S_{G_{\lambda} w}{ }^{-1}\left(B\left(A_{G_{\lambda} w}{ }^{-1}(t)\right)\right), \quad t \geq 0 .
\end{aligned}
\end{aligned}
$$

Here $S_{G_{\lambda} w}{ }^{-1}$ and $A_{G_{\lambda} w}{ }^{-1}$ denote the inverse functions of $S_{G_{\lambda} w}$ and $A_{G_{\lambda} w}$, respectively. For $x_{0} \in \mathbb{R}$, define $\left(G_{\lambda} w\right)^{x_{0}} \in \widetilde{\mathbb{W}}$ by $\left(G_{\lambda} w\right)^{x_{0}}(x)=\left(G_{\lambda} w\right)\left(x+x_{0}\right), x \in \mathbb{R}$, and set

$$
X\left(t ; x_{0}, G_{\lambda} w\right)=x_{0}+X\left(t ; 0,\left(G_{\lambda} w\right)^{x_{0}}\right), \quad t \geq 0 .
$$

Then, on $(\widetilde{\Omega}, \widetilde{P})$, we get a diffusion process $\left\{X\left(t ; x_{0}, G_{\lambda} w\right), t \geq 0\right\}$ starting from $x_{0}$ whose generator is $\mathcal{L}_{G_{\lambda} w}$.

LEMMA 2.1. For any $w \in \mathbb{W}$ and $\lambda>0$

$$
\left\{X(t), t \geq 0, P_{G_{\lambda}\left(\tau_{\lambda} w\right)}^{0}\right\} \stackrel{\mathrm{d}}{=}\left\{e^{-a \lambda} X\left(e^{2 a \lambda} t\right), t \geq 0, P_{T_{\lambda} w}^{0}\right\} .
$$

Proof. We can prove the lemma in the same way as in [6] (see also [1]) by using the equality

$$
\left(G_{\lambda}\left(\tau_{\lambda} w\right)\right)(x)=\left(T_{\lambda} w\right)\left(e^{a \lambda} x\right), \quad x \in \mathbb{R} .
$$

Owing to Lemma 2.1 and (1.2), we obtain Theorem 1.1 from the following proposition by the same argument as in [8, p. 531] (see also [3]).

Proposition 2.2. (i) There exists a subset $\mathbb{A}^{\#}$ of $\mathbb{A}$ with $P\left\{\mathbb{A} \backslash \mathbb{A}^{\#}\right\}=0$ such that, for any $w \in \mathbb{A}^{\#}$ and $\varepsilon>0$

$$
\lim _{\lambda \rightarrow \infty} P_{G_{\lambda} w}^{0}\left\{b-\varepsilon<\lambda^{-2} e^{a \lambda} X\left(e^{\lambda(1-2 a)}\right)<(b+\varepsilon) \wedge 0\right\}=1 .
$$

(ii) There exists a subset $\mathbb{B}^{\#}$ of $\mathbb{B}$ with $P\left\{\mathbb{B} \backslash \mathbb{B}^{\#}\right\}=0$ such that, for any $w \in \mathbb{B}^{\#}$

$$
\lim _{\lambda \rightarrow \infty} P_{G_{\lambda} w}^{0}\left\{0<X\left(e^{\lambda(1-2 a)}\right)<1\right\}=1 .
$$



$\varepsilon>0$

(iii) There exists a subset $\mathbb{C}^{\#}$ of $\mathbb{C}$ with $P\left\{\mathbb{C} \backslash \mathbb{C}^{\#}\right\}=0$ such that, for any $w \in \mathbb{C}^{\#}$ and

$$
\lim _{\lambda \rightarrow \infty} P_{G_{\lambda} w}^{0}\left\{-\left(\exp \left\{e^{a \lambda / 2}(w(1)+\varepsilon)\right\} \wedge \varepsilon\right)<X\left(e^{\lambda(1-2 a)}\right)-1<\varepsilon\right\}=1 .
$$

In Section 3 we present lemmas on hitting times of the diffusion process constructed in Section 2. In Section 4 we prove Proposition 2.2. In Section 5 we show Theorem 1.3 and in Section 6 we show Theorem 1.4.

\section{Estimation on hitting times}

In this section we estimate hitting times of the diffusion process introduced in Section 2 by improving (or using) the method in [8] ([1], [4]). For $\omega \in \Omega$, we set

$$
\tau(q)=\tau(q, \omega)=\inf \{t>0: X(t)=q\}, \quad q \in \mathbb{R} .
$$

In the following lemma which is used to prove Theorem 1.4, we have more precise estimation on a hitting time from above than that in [8] ([4]).

LEMMA 3.1. Let $w \in \mathbb{W}$ and $p \leq p_{\lambda}<x_{0} \leq 0$ for all sufficiently large $\lambda>0$. Assume $w\left(p_{\lambda}\right) \geq w(x)$ for all $x \in\left[p_{\lambda}, x_{0}\right]$ for all sufficiently large $\lambda>0$. In addition, assume (i) $\max _{p \leq x \leq 0} w(x)<a$ or (ii) $w(1)>0$. Then for some $C>0$

$$
\lim _{\lambda \rightarrow \infty} P_{G_{\lambda} w}^{\lambda^{2} e^{-a \lambda} x_{0}}\left\{\tau\left(\lambda^{2} e^{-a \lambda} p_{\lambda}\right)<C e^{\lambda J(\lambda)}\right\}=1
$$

where

$$
\begin{aligned}
& J(\lambda)=\max \left\{w\left(p_{\lambda}\right)-V_{\lambda}-2 a+4 \lambda^{-1} \log \lambda+\lambda^{-1} \log \log \lambda,\right. \\
& \left.w\left(p_{\lambda}\right)-a+2 \lambda^{-1} \log \lambda+\lambda^{-1} \log \log \lambda\right\}, \\
& V_{\lambda}=\min _{p_{\lambda} \leq x \leq 0} w(x) .
\end{aligned}
$$

PROOF. We set

$$
\begin{aligned}
& \tau\left(q ; x_{0}, G_{\lambda} w\right)=\inf \left\{t>0: X\left(t ; x_{0}, G_{\lambda} w\right)=q\right\}, \quad q \in \mathbb{R}, \\
& T(q)=\inf \{t>0: B(t)=q\}, \quad q \in \mathbb{R},
\end{aligned}
$$

which are defined on the probability space $(\widetilde{\Omega}, \widetilde{P})$. The assertion $(3.1)$ is equivalent to

$$
\lim _{\lambda \rightarrow \infty} \widetilde{P}\left\{\tau\left(\lambda^{2} e^{-a \lambda} p_{\lambda} ; \lambda^{2} e^{-a \lambda} x_{0}, G_{\lambda} w\right)<C e^{\lambda J(\lambda)}\right\}=1 .
$$

We just prove (3.2) in the case $x_{0}=0$ and the assumption (i) is satisfied. We set

$$
E_{\lambda}=\left\{\tau\left(\lambda^{2} e^{-a \lambda} p_{\lambda} ; 0, G_{\lambda} w\right)<\tau\left(1 ; 0, G_{\lambda} w\right)\right\} .
$$

By the assumption (i), we have

$$
\lim _{\lambda \rightarrow \infty} \widetilde{P}\left\{\tau\left(\lambda^{2} e^{-a \lambda} p ; 0, G_{\lambda} w\right)<\tau\left(1 ; 0, G_{\lambda} w\right)\right\}=1
$$


and therefore

$$
\lim _{\lambda \rightarrow \infty} \widetilde{P}\left\{E_{\lambda}\right\}=1
$$

By (2.2) and (2.1), we observe

$$
\begin{aligned}
\tau\left(\lambda^{2} e^{-a \lambda} p_{\lambda} ; 0, G_{\lambda} w\right) & =A_{G_{\lambda} w}\left(T\left(S_{G_{\lambda} w}\left(\lambda^{2} e^{-a \lambda} p_{\lambda}\right)\right)\right) \\
& =\int_{\lambda^{2} e^{-a \lambda} p_{\lambda}}^{\infty} e^{-\left(G_{\lambda} w\right)(x)} L\left(T\left(S_{G_{\lambda} w}\left(\lambda^{2} e^{-a \lambda} p_{\lambda}\right)\right), S_{G_{\lambda} w}(x)\right) d x .
\end{aligned}
$$

On the set $E_{\lambda}$, the right-hand side of (3.4) equals

$$
\begin{aligned}
& \int_{\lambda^{2} e^{-a \lambda} p_{\lambda}}^{1} e^{-\left(G_{\lambda} w\right)(x)} L\left(T\left(S_{G_{\lambda} w}\left(\lambda^{2} e^{-a \lambda} p_{\lambda}\right)\right), S_{G_{\lambda} w}(x)\right) d x \\
& \stackrel{\mathrm{d}}{=}\left|S_{G_{\lambda} w}\left(\lambda^{2} e^{-a \lambda} p_{\lambda}\right)\right| \int_{\lambda^{2} e^{-a \lambda} p_{\lambda}}^{1} e^{-\left(G_{\lambda} w\right)(x)} L\left(T(-1), \frac{S_{G_{\lambda} w}(x)}{\left|S_{G_{\lambda} w}\left(\lambda^{2} e^{-a \lambda} p_{\lambda}\right)\right|}\right) d x \\
& =\lambda^{4} e^{-2 a \lambda} \int_{p_{\lambda}}^{0} e^{\lambda w(y)} d y \int_{p_{\lambda}}^{0} e^{-\lambda w(z)} L\left(T(-1), \frac{S_{G_{\lambda} w}\left(\lambda^{2} e^{-a \lambda} z\right)}{\left|S_{G_{\lambda} w}\left(\lambda^{2} e^{-a \lambda} p_{\lambda}\right)\right|} d z\right. \\
& \quad+\lambda^{2} e^{-a \lambda} \int_{p_{\lambda}}^{0} e^{\lambda w(y)} d y \int_{0}^{1} L\left(T(-1), \frac{S_{G_{\lambda} w}(z)}{\left|S_{G_{\lambda} w}\left(\lambda^{2} e^{-a \lambda} p_{\lambda}\right)\right|}\right) d z \\
& \equiv I_{\lambda}+I I_{\lambda} .
\end{aligned}
$$

For $t>0$, we set $K(t)=\sup _{x \in \mathbb{R}} L(t, x)$. Since $0<K(T(-1))<\infty(\widetilde{P}$-a.s. $)$, we have for all sufficiently large $\lambda>0$

$$
\begin{aligned}
& I_{\lambda} \leq|p|^{2} K(T(-1)) e^{\lambda\left(w\left(p_{\lambda}\right)-V_{\lambda}-2 a+4 \lambda^{-1} \log \lambda\right)}, \quad \widetilde{P} \text {-a.s. } \\
& I I_{\lambda} \leq|p| K(T(-1)) e^{\lambda\left(w\left(p_{\lambda}\right)-a+2 \lambda^{-1} \log \lambda\right)}, \quad \widetilde{P} \text {-a.s. }
\end{aligned}
$$

Set $E_{\lambda}^{\prime}=\{K(T(-1))<\log \lambda\}$. Then we have $\lim _{\lambda \rightarrow \infty} \widetilde{P}\left\{E_{\lambda}^{\prime}\right\}=1$, and for all sufficiently large $\lambda>0$ the following holds on $E_{\lambda}^{\prime}$ :

$$
I_{\lambda}+I I_{\lambda}<\left(|p|^{2}+|p|\right) e^{\lambda J(\lambda)} .
$$

Therefore we have

$$
\lim _{\lambda \rightarrow \infty} \widetilde{P}\left\{I_{\lambda}+I I_{\lambda}<\left(|p|^{2}+|p|\right) e^{\lambda J(\lambda)}\right\}=1 .
$$

By (3.3)-(3.6), we obtain (3.2) in the case $x_{0}=0$.

The following lemma is easily obtained from Lemma 3.1.

Lemma 3.2. Let $w \in \mathbb{W}$ and $p<x_{0} \leq 0$. Assume $w(p) \geq w(x)$ for all $x \in\left[p, x_{0}\right]$. In addition, assume (i) $\max _{p \leq x \leq 0} w(x)<$ a or (ii) $w(1)>0$. Then for any $\varepsilon>0$

$$
\lim _{\lambda \rightarrow \infty} P_{G_{\lambda} w}^{\lambda^{2} e^{-a \lambda} x_{0}}\left\{\tau\left(\lambda^{2} e^{-a \lambda} p\right)<e^{\lambda\left(J_{I}+\varepsilon\right)}\right\}=1,
$$


where

$$
\begin{aligned}
J_{I} & =\max \left\{J_{0}-2 a, w(p)-a\right\} \\
& = \begin{cases}J_{0}-2 a, & \text { if } \min _{p \leq x \leq 0} w(x) \leq-a, \\
w(p)-a, & \text { if } \min _{p \leq x \leq 0} w(x) \geq-a,\end{cases} \\
J_{0} & =w(p)-\min _{p \leq x \leq 0} w(x) .
\end{aligned}
$$

LEMMA 3.3. Let $w \in \mathbb{W}$ and $p<x_{0} \leq 0$.

(i) Assume $w(p)>w(x)$ for all $x \in\left(p, x_{0}\right]$. Then for any $\varepsilon>0$

$$
\lim _{\lambda \rightarrow \infty} P_{G_{\lambda} w}^{\lambda^{2} e^{-a \lambda} x_{0}}\left\{\tau\left(\lambda^{2} e^{-a \lambda} p\right)>e^{\lambda\left(J_{x_{0}}-2 a-\varepsilon\right)}\right\}=1,
$$

where $J_{x_{0}}=w(p)-\min _{p \leq x \leq x_{0}} w(x)$.

(ii) Assume $w(p)>w(x)$ for all $x \in(p, 0]$ and $w(p)>a$. Then for any $\varepsilon>0$

$$
\lim _{\lambda \rightarrow \infty} P_{G_{\lambda} w}^{0}\left\{\tau\left(\lambda^{2} e^{-a \lambda} p\right)>e^{\lambda\left(J_{I}-\varepsilon\right)}\right\}=1,
$$

where $J_{I}$ is defined in (3.8).

Proof. We just prove (ii). We show

$$
\lim _{\lambda \rightarrow \infty} \widetilde{P}\left\{\tau\left(\lambda^{2} e^{-a \lambda} p ; 0, G_{\lambda} w\right)>e^{\lambda\left(J_{I}-\varepsilon\right)}\right\}=1,
$$

which is equivalent to (3.9). As in the proof of Lemma 3.1, we have

$$
\begin{aligned}
\tau\left(\lambda^{2} e^{-a \lambda} p ; 0, G_{\lambda} w\right) & \stackrel{\mathrm{d}}{=}\left|S_{G_{\lambda} w}\left(\lambda^{2} e^{-a \lambda} p\right)\right| \int_{\lambda^{2} e^{-a \lambda} p}^{\infty} e^{-\left(G_{\lambda} w\right)(x)} L\left(T(-1), \frac{S_{G_{\lambda} w}(x)}{\left|S_{G_{\lambda} w}\left(\lambda^{2} e^{-a \lambda} p\right)\right|}\right) d x \\
\geq & \left|S_{G_{\lambda} w}\left(\lambda^{2} e^{-a \lambda} p\right)\right| \int_{\lambda^{2} e^{-a \lambda} p}^{1} e^{-\left(G_{\lambda} w\right)(x)} L\left(T(-1), \frac{S_{G_{\lambda} w}(x)}{\left|S_{G_{\lambda} w}\left(\lambda^{2} e^{-a \lambda} p\right)\right|}\right) d x \\
= & \lambda^{4} e^{-2 a \lambda} \int_{p}^{0} e^{\lambda w(y)} d y \int_{p}^{0} e^{-\lambda w(z)} L\left(T(-1), \frac{S_{G_{\lambda} w}\left(\lambda^{2} e^{-a \lambda} z\right)}{\left|S_{G_{\lambda} w}\left(\lambda^{2} e^{-a \lambda} p\right)\right|}\right) d z \\
& +\lambda^{2} e^{-a \lambda} \int_{p}^{0} e^{\lambda w(y)} d y \int_{0}^{1} L\left(T(-1), \frac{S_{G_{\lambda} w}(z)}{\left|S_{G_{\lambda} w}\left(\lambda^{2} e^{-a \lambda} p\right)\right|}\right) d z \\
\equiv & I I I_{\lambda}+I V_{\lambda} .
\end{aligned}
$$

First we estimate $I I I_{\lambda}$. We observe that

$$
\frac{\left|S_{G_{\lambda} w}\left(\lambda^{2} e^{-a \lambda} z\right)\right|}{\left|S_{G_{\lambda} w}\left(\lambda^{2} e^{-a \lambda} p\right)\right|}=\frac{\int_{z}^{0} e^{\lambda w(u)} d u}{\int_{p}^{0} e^{\lambda w(u)} d u} \rightarrow 0
$$


as $\lambda \rightarrow \infty$ uniformly on any closed interval contained in $(p, 0]$. From this, it follows that

$$
L\left(T(-1), \frac{S_{G_{\lambda} w}\left(\lambda^{2} e^{-a \lambda} z\right)}{\left|S_{G_{\lambda} w}\left(\lambda^{2} e^{-a \lambda} p\right)\right|}\right) \rightarrow L(T(-1), 0)>0 \quad(\widetilde{P} \text {-a.s. })
$$

as $\lambda \rightarrow \infty$ uniformly on any closed interval contained in $(p, 0]$. Therefore, by virtue of the classical Laplace method, we have

$$
\lim _{\lambda \rightarrow \infty} \frac{1}{\lambda} \log I I I_{\lambda}=J_{0}-2 a, \quad \widetilde{P} \text {-a.s. }
$$

Next we estimate $I V_{\lambda}$. Since

$$
\frac{S_{G_{\lambda} w}(z)}{\left|S_{G_{\lambda} w}\left(\lambda^{2} e^{-a \lambda} p\right)\right|}=\frac{e^{a \lambda} z}{\lambda^{2} \int_{p}^{0} e^{\lambda w(u)} d u} \rightarrow 0
$$

as $\lambda \rightarrow \infty$ uniformly on any closed interval contained in $(0,1)$, we get

$$
\lim _{\lambda \rightarrow \infty} \frac{1}{\lambda} \log I V_{\lambda}=w(p)-a, \quad \widetilde{P} \text {-a.s. }
$$

in the same way as above. By (3.12) and (3.13), we obtain

$$
\lim _{\lambda \rightarrow \infty} \frac{1}{\lambda} \log \left(I I I_{\lambda}+I V_{\lambda}\right)=J_{I}
$$

in probability with respect to $\widetilde{P}$. Therefore, by (3.11), we arrive at (3.10).

LEMmA 3.4. Let $w \in \mathbb{W}$ and assume $\sigma(a)>-\infty$. Then for any $\varepsilon>0$

$$
\lim _{\lambda \rightarrow \infty} P_{G_{\lambda} w}^{0}\left\{\tau(1)<e^{\lambda\left(J_{I I}+\varepsilon\right)}\right\}=1,
$$

where $J_{I I}=\max \left\{-\min _{\sigma(a) \leq x \leq 0} w(x)-a, 0\right\}$.

ProOF. We can prove the lemma by following the proof of Lemma 3.1 and using

$$
\lim _{\lambda \rightarrow \infty} P_{G_{\lambda} w}^{0}\left\{\tau(1)<\tau\left(\lambda^{2} e^{-a \lambda} \sigma(a)\right)\right\}=1
$$

instead of (3.3).

The following lemma can be shown in the same way as Lemma 3.3.

Lemma 3.5. Let $w \in \mathbb{W}$ and $p>1$. Assume $w(p)>w(x)$ for all $x \in[1, p)$. Then for any $\varepsilon>0$

$$
\lim _{\lambda \rightarrow \infty} P_{G_{\lambda} w}^{1}\left\{\tau(p)>\exp \left\{e^{a \lambda / 2}\left(J_{I I I}-\varepsilon\right)\right\}\right\}=1,
$$

where $J_{I I I}=w(p)-\min _{1 \leq x \leq p} w(x)$. 


\section{Proof of Proposition 2.2}

First we prepare two lemmas which are used to prove Proposition 2.2 (i). We show these lemmas by using the method in [8] (see also [3], [4]).

LEMma 4.1. There exists a subset $\mathbb{A}^{\#}$ of $\mathbb{A}$ with $P\left\{\mathbb{A} \backslash \mathbb{A}^{\#}\right\}=0$ such that for any $w \in \mathbb{A}^{\#}$ the following holds: for any sufficiently small $u>0$ there exists $\delta_{0}>0$ such that for any $\delta \in\left(0, \delta_{0}\right)$

$$
\lim _{\lambda \rightarrow \infty} P_{G_{\lambda} w}^{0}\left\{\tau\left(\lambda^{2} e^{-a \lambda} \zeta_{1-u}\right)<e^{\lambda(1-2 a-\delta)}\right\}=1 .
$$

Proof. Let $w \in \mathbb{A}$. In the case $M<V+1$, we let $u>0$ satisfy $w\left(\zeta_{1-u}\right)>w(x)$ for all $x \in\left(\zeta_{1-u}, 0\right]$. Then we can apply Lemma 3.2 to $p=\zeta_{1-u}$ and $x_{0}=0$ because of (1.6) or the definition of $\mathbb{A}^{\prime \prime}$. In this case the assertion (3.7) holds for $J_{I}=w\left(\zeta_{1-u}\right)-$ $\min _{\zeta_{1-u} \leq x \leq 0} w(x)-2 a=1-u-2 a$ because of (1.1). As a result, we get (4.1) for any $\delta \in(0, u)$.

In the case $M \geq V+1$, we let $u \in(0,1)$ and set $c_{0}=0$. For some integer $n \geq 2$ we take $\ell_{k}<c_{k}<0, k \in\{1,2, \ldots, n\}$, satisfying $\sigma(-a)=c_{1}>c_{2}>\cdots>c_{n-1}>b>c_{n}=\zeta_{1-u}$ and

$$
\left\{\begin{array}{l}
w_{k}\left(\ell_{k}\right) \geq w_{k}(x) \quad \text { for all } x \in\left[\ell_{k}, c_{k-1}\right] \\
w_{k}\left(\ell_{k}\right)<a \text { if } w \in \mathbb{A}^{\prime} \\
w_{k}\left(\ell_{k}\right)-\min _{c_{k} \leq x \leq 0} w_{k}(x)<1
\end{array}\right.
$$

for any $k \in\{1,2, \ldots, n\}$, where $w_{k} \in \mathbb{W}$ is defined by

$$
w_{k}(x)= \begin{cases}w(x) & \text { for } x \geq c_{k}, \\ -x+w\left(c_{k}\right)+c_{k} & \text { for } x<c_{k} .\end{cases}
$$

Note that we can take $c_{k}, k \in\{1,2, \ldots, n-1\}$, independent of $u$. By (4.2) and Lemma 3.2, we have, for any $k \in\{1,2, \ldots, n\}$ and $\varepsilon_{k}>0$

$$
\lim _{\lambda \rightarrow \infty} P_{G_{\lambda} w_{k}}^{\lambda^{2} e^{-a \lambda} c_{k-1}}\left\{\tau\left(\lambda^{2} e^{-a \lambda} \ell_{k}\right)<e^{\lambda\left(\bar{J}_{k}-2 a+\varepsilon_{k}\right)}\right\}=1,
$$

where $\bar{J}_{k}=w_{k}\left(\ell_{k}\right)-\min _{\ell_{k} \leq x \leq 0} w_{k}(x)<1$. Therefore, for any $k \in\{1,2, \ldots, n\}$ and $\delta_{k} \in\left(0,1-\bar{J}_{k}\right)$, we have

$$
\lim _{\lambda \rightarrow \infty} P_{G_{\lambda} w}^{\lambda^{2} e^{-a \lambda} c_{k-1}}\left\{\tau\left(\lambda^{2} e^{-a \lambda} c_{k}\right)<e^{\lambda\left(1-2 a-\delta_{k}\right)}\right\}=1 .
$$

Using the strong Markov property, we obtain the lemma in this case, too.

LEMMA 4.2. There exists a subset $\mathbb{A}^{\#}$ of $\mathbb{A}$ with $P\left\{\mathbb{A} \backslash \mathbb{A}^{\#}\right\}=0$ such that for any $w \in \mathbb{A}^{\#}$ the following holds: for any $v>0$ satisfying $\min _{\zeta_{1+v} \leq x \leq \zeta} w(x)>V$ and any $\delta \in(0, v)$

$$
\lim _{\lambda \rightarrow \infty} P_{G_{\lambda} w}^{0}\left\{\tau\left(\lambda^{2} e^{-a \lambda} \zeta_{1+v}\right)>e^{\lambda(1-2 a+\delta)}\right\}=1
$$


PRoOF. Let $w \in \mathbb{A}$ and $v>0$ satisfy $\min _{\zeta_{1+v} \leq x \leq \zeta} w(x)>V$. In the case $M<V+1$, we can apply Lemma 3.3 (i) to $p=\zeta_{1+v}$ and $x_{0}=0$, and because of

$$
\bar{J} \equiv w\left(\zeta_{1+v}\right)-\min _{\zeta_{1+v} \leq x \leq 0} w(x)=1+v,
$$

we get (4.3) for any $\delta \in(0, v)$. We show (4.3) in the case $M \geq V+1$. For $c_{n-1}<0$ defined in the proof of Lemma 4.1, we have, for any sufficiently small $\bar{\delta}>0$

$$
\lim _{\lambda \rightarrow \infty} P_{G_{\lambda} w}^{0}\left\{\tau\left(\lambda^{2} e^{-a \lambda} c_{n-1}\right)<e^{\lambda(1-2 a-\bar{\delta})}\right\}=1 .
$$

We note that $w\left(\zeta_{1+v}\right)>w(x)$ for all $x \in\left(\zeta_{1+v}, c_{n-1}\right]$. Therefore, by Lemma 3.3 (i), we have, for any $\varepsilon>0$

$$
\lim _{\lambda \rightarrow \infty} P_{G_{\lambda} w}^{\lambda^{2} e^{-a \lambda} c_{n-1}}\left\{\tau\left(\lambda^{2} e^{-a \lambda} \zeta_{1+v}\right)>e^{\lambda(\bar{J}-2 a-\varepsilon)}\right\}=1 .
$$

By (4.5), (4.6), (4.4) and the strong Markov property, we obtain (4.3) for any $\delta \in(0, v)$ in this case, too.

Let us now prove Proposition 2.2. To prove (i) and (ii), we use the coupling method in [6] (see also [8]).

Proof of Proposition 2.2. First we show (i). Let $w \in \mathbb{A}$ and set

$$
r= \begin{cases}1 & \text { if } w \in \mathbb{A}^{\prime} \\ 1+\eta_{1} & \text { if } w \in \mathbb{A}^{\prime \prime} \cap\left(\mathbb{A}^{\prime}\right)^{c} .\end{cases}
$$

In the case $w \in \mathbb{A}^{\prime \prime} \cap\left(\mathbb{A}^{\prime}\right)^{c}, \eta_{1}>0$ is chosen to be small enough that $C\left(\eta_{1}\right) \equiv$ $\min _{1 \leq x \leq 1+\eta_{1}} w(x)>0$. Let $v>0$ satisfy

$$
\min _{\zeta_{1+v} \leq x \leq \zeta} w(x)>V
$$

and

$$
w\left(\zeta_{1+v}\right)<a \quad \text { if } w \in \mathbb{A}^{\prime}
$$

Then we have

$$
\lim _{\lambda \rightarrow \infty} P_{G_{\lambda} w}^{0}\left\{\tau(r)>\tau\left(\lambda^{2} e^{-a \lambda} \zeta_{1+v}\right)\right\}=1
$$

because of (1.6) and (4.8) (in the case $w \in \mathbb{A}^{\prime}$ ). We set $K_{\lambda}=\left[\lambda^{2} e^{-a \lambda} \zeta_{1+v}, r\right]$ and define the probability measure $m_{\lambda}$ on $K_{\lambda}$ by

$$
m_{\lambda}(d x)=\frac{e^{-\left(G_{\lambda} w\right)(x)} d x}{\int_{K_{\lambda}} e^{-\left(G_{\lambda} w\right)(y)} d y} .
$$


This is the invariant probability measure for the reflecting $\mathcal{L}_{G_{\lambda} w}$-diffusion process on $K_{\lambda}$. Let $\varepsilon \in(0, b-\zeta)$. In the case $w \in \mathbb{A}^{\prime \prime} \cap\left(\mathbb{A}^{\prime}\right)^{c}$ we have

$$
\begin{aligned}
m_{\lambda}\left\{\left(\lambda^{2} e^{-a \lambda}(b-\varepsilon), \lambda^{2} e^{-a \lambda}(b+\varepsilon) \wedge 0\right)\right\} & \\
= & \frac{\int_{b-\varepsilon}^{(b+\varepsilon) \wedge 0} e^{-\lambda w(y)} d y}{\int_{\zeta_{1+v}}^{0} e^{-\lambda w(y)} d y+\lambda^{-2} e^{a \lambda}+\lambda^{-2} e^{a \lambda} \int_{1}^{1+\eta_{1}} \exp \left\{-e^{a \lambda / 2} w(y)\right\} d y} .
\end{aligned}
$$

By virtue of (1.1) and (4.7), we have

$$
\begin{aligned}
& \lim _{\lambda \rightarrow \infty} \lambda^{-1} \log \int_{b-\varepsilon}^{(b+\varepsilon) \wedge 0} e^{-\lambda w(y)} d y=-V>a, \\
& \lim _{\lambda \rightarrow \infty} \lambda^{-1} \log \int_{\left(\zeta_{1+v}, 0\right) \backslash(b-\varepsilon,(b+\varepsilon) \wedge 0)} e^{-\lambda w(y)} d y<-V, \\
& \lim _{\lambda \rightarrow \infty} e^{-a \lambda / 2} \log \int_{1}^{1+\eta_{1}} \exp \left\{-e^{a \lambda / 2} w(y)\right\} d y=-C\left(\eta_{1}\right)<0 .
\end{aligned}
$$

Therefore we get

$$
\lim _{\lambda \rightarrow \infty} m_{\lambda}\left\{\left(\lambda^{2} e^{-a \lambda}(b-\varepsilon), \lambda^{2} e^{-a \lambda}(b+\varepsilon) \wedge 0\right)\right\}=1
$$

in the case $w \in \mathbb{A}^{\prime \prime} \cap\left(\mathbb{A}^{\prime}\right)^{c}$. As easily seen from above, we get (4.10) in the case $w \in \mathbb{A}^{\prime}$, too.

We introduce $\left\{X_{\lambda}^{(R)}(t), t \geq 0\right\}$, the reflecting $\mathcal{L}_{G_{\lambda} w}$-diffusion process on $K_{\lambda}$ with initial distribution $m_{\lambda}$ defined on the probability space $(\widetilde{\Omega}, \widetilde{P})$. Since this is a stationary process, we have, by (4.10), for any $t \geq 0$

$$
\lim _{\lambda \rightarrow \infty} \widetilde{P}\left\{X_{\lambda}^{(R)}(t) \in\left(\lambda^{2} e^{-a \lambda}(b-\varepsilon), \lambda^{2} e^{-a \lambda}(b+\varepsilon) \wedge 0\right)\right\}=1 .
$$

We couple the processes $\left\{X\left(t ; 0, G_{\lambda} w\right), t \geq 0\right\}$ and $\left\{X_{\lambda}^{(R)}(t), t \geq 0\right\}$ as follows: two processes move independently until they first meet each other; then they move together until they go out from $\left(\lambda^{2} e^{-a \lambda} \zeta_{1+v}, r\right)$; after going out from the interval they again move independently. Let

$$
\begin{aligned}
& \sigma_{\lambda}=\inf \left\{t>0: X\left(t ; 0, G_{\lambda} w\right)=X_{\lambda}^{(R)}(t)\right\}, \\
& \sigma_{\lambda}^{\prime}=\inf \left\{t>\sigma_{\lambda}: X\left(t ; 0, G_{\lambda} w\right) \notin\left(\lambda^{2} e^{-a \lambda} \zeta_{1+v}, r\right)\right\} .
\end{aligned}
$$

By (4.11), it follows that

$$
\lim _{\lambda \rightarrow \infty} \widetilde{P}\left\{\sigma_{\lambda}<\tau\left(\lambda^{2} e^{-a \lambda}(b-\varepsilon) ; 0, G_{\lambda} w\right)\right\}=1 .
$$

By using (4.12) and Lemma 4.1 for sufficiently small $u>0$ satisfying $\zeta_{1-u}<b-\varepsilon$, we have, for any sufficiently small $\delta>0$

$$
\lim _{\lambda \rightarrow \infty} \widetilde{P}\left\{\sigma_{\lambda}<e^{\lambda(1-2 a-\delta)}\right\}=1 .
$$


Moreover, by virtue of (4.9) and Lemma 4.2, we have, for any $\delta^{\prime} \in(0, v)$

$$
\lim _{\lambda \rightarrow \infty} \widetilde{P}\left\{\sigma_{\lambda}^{\prime}>e^{\lambda\left(1-2 a+\delta^{\prime}\right)}\right\}=1 .
$$

Using (4.13), (4.14) and (4.11), we obtain, for any $\varepsilon \in(0, b-\zeta)$

$$
\lim _{\lambda \rightarrow \infty} \widetilde{P}\left\{X\left(e^{\lambda(1-2 a)} ; 0, G_{\lambda} w\right) \in\left(\lambda^{2} e^{-a \lambda}(b-\varepsilon), \lambda^{2} e^{-a \lambda}(b+\varepsilon) \wedge 0\right)\right\}=1
$$

by the same argument as in [6] (see also [8]). Therefore we get (i).

Next we prove (ii). Let $w \in \mathbb{B}$. In this case, in Lemma 3.4 we have $J_{I I}=0$ and therefore for any $\varepsilon>0$

$$
\lim _{\lambda \rightarrow \infty} P_{G_{\lambda} w}^{0}\left\{\tau(1)<e^{\lambda \varepsilon}\right\}=1 .
$$

We set $q=\sigma(1-a+\xi)$, where $\xi>0$ is chosen to be small enough that $\min _{q \leq x \leq \sigma(1-a)} w(x)>-a$. Then we have $\widetilde{V} \equiv \min _{q \leq x \leq 0} w(x)>-a$. Applying Lemma 3.3 (ii) to $p=q$, we have, for any $\widetilde{\delta} \in(0, \xi)$

$$
\lim _{\lambda \rightarrow \infty} P_{G_{\lambda} w}^{0}\left\{\tau\left(\lambda^{2} e^{-a \lambda} q\right)>e^{\lambda(1-2 a+\widetilde{\delta})}\right\}=1 .
$$

Moreover, for any $\eta>0$, we see that

$$
\lim _{\lambda \rightarrow \infty} P_{G_{\lambda} w}^{0}\left\{\tau(1+\eta)>\tau\left(\lambda^{2} e^{-a \lambda} q\right)\right\}=1 .
$$

Choose $\eta_{2}>0$ satisfying $C\left(\eta_{2}\right) \equiv \min _{1 \leq x \leq 1+\eta_{2}} w(x)>0$, and set $\widetilde{K}_{\lambda}=\left[\lambda^{2} e^{-a \lambda} q, 1+\eta_{2}\right]$. We define $\widetilde{m}_{\lambda}$, a probability measure on $\widetilde{K}_{\lambda}$, by

$$
\tilde{m}_{\lambda}(d x)=\frac{e^{-\left(G_{\lambda} w\right)(x)} d x}{\int_{\widetilde{K}_{\lambda}} e^{-\left(G_{\lambda} w\right)(y)} d y} .
$$

We observe

$$
\begin{aligned}
\tilde{m}_{\lambda}\{(0,1)\} & =\frac{1}{\lambda^{2} e^{-a \lambda} \int_{q}^{0} e^{-\lambda w(y)} d y+1+\int_{1}^{1+\eta_{2}} \exp \left\{-e^{a \lambda / 2} w(y)\right\} d y} \\
& \rightarrow 1, \quad \text { as } \lambda \rightarrow \infty,
\end{aligned}
$$

since

$$
\begin{aligned}
& \lim _{\lambda \rightarrow \infty} \lambda^{-1} \log \int_{q}^{0} e^{-\lambda w(y)} d y=-\widetilde{V}<a, \\
& \lim _{\lambda \rightarrow \infty} e^{-a \lambda / 2} \log \int_{1}^{1+\eta_{2}} \exp \left\{-e^{a \lambda / 2} w(y)\right\} d y=-C\left(\eta_{2}\right)<0 .
\end{aligned}
$$

Let $\left\{\widetilde{X}_{\lambda}^{(R)}(t), t \geq 0\right\}$ be the reflecting $\mathcal{L}_{G_{\lambda} w}$-diffusion process on $\widetilde{K}_{\lambda}$ with initial distribution $\tilde{m}_{\lambda}$ defined on $(\widetilde{\Omega}, \widetilde{P})$. We couple $\left\{X\left(t ; 0, G_{\lambda} w\right), t \geq 0\right\}$ and $\left\{\widetilde{X}_{\lambda}^{(R)}(t), t \geq 0\right\}$ in the same 
way as we coupled $\left\{X\left(t ; 0, G_{\lambda} w\right), t \geq 0\right\}$ and $\left\{X_{\lambda}^{(R)}(t), t \geq 0\right\}$ in the proof of (i). By the same argument as there and using (4.15)-(4.18), we obtain (ii).

Finally we prove (iii). Let $w \in \mathbb{C}$. In this case, in Lemma 3.4 we have $0 \leq J_{I I}<1-2 a$ and therefore for any $\delta \in\left(0,1-2 a-J_{I I}\right)$

$$
\lim _{\lambda \rightarrow \infty} P_{G_{\lambda} w}^{0}\left\{\tau(1)<e^{\lambda(1-2 a-\delta)}\right\}=1 .
$$

By Lemma 3.5, for any $\varepsilon>0$ there exists $C^{\prime}>0$ such that

$$
\lim _{\lambda \rightarrow \infty} P_{G_{\lambda} w}^{1}\left\{\tau(1+\varepsilon)>\exp \left\{e^{a \lambda / 2} C^{\prime}\right\}\right\}=1
$$

By (4.19) and (4.20), we get, for any $\varepsilon>0$

$$
\lim _{\lambda \rightarrow \infty} P_{G_{\lambda} w}^{0}\left\{X\left(e^{\lambda(1-2 a)}\right)<1+\varepsilon\right\}=1 .
$$

On the other hand, we let $\varepsilon \in(0,-w(1))$ and choose $\eta_{3}>0$ satisfying $M\left(\eta_{3}\right) \equiv$ $\max _{1 \leq x \leq 1+\eta_{3}} w(x)<w(1)+\varepsilon(<0)$. We observe

$$
\begin{aligned}
P_{G_{\lambda} w}^{1} & \left\{\tau\left(1-\exp \left\{e^{a \lambda / 2}(w(1)+\varepsilon)\right\}\right)>\tau\left(1+\eta_{3}\right)\right\} \\
= & \frac{\exp \left\{e^{a \lambda / 2}(w(1)+\varepsilon)\right\}}{\int_{1}^{1+\eta_{3}} \exp \left\{e^{a \lambda / 2} w(x)\right\} d x+\exp \left\{e^{a \lambda / 2}(w(1)+\varepsilon)\right\}} \\
& \rightarrow 1, \quad \text { as } \lambda \rightarrow \infty,
\end{aligned}
$$

since

$$
\lim _{\lambda \rightarrow \infty} e^{-a \lambda / 2} \log \int_{1}^{1+\eta_{3}} \exp \left\{e^{a \lambda / 2} w(x)\right\} d x=M\left(\eta_{3}\right)<w(1)+\varepsilon .
$$

Using (4.22) and Lemma 3.5, we have for some $C^{\prime \prime}>0$

$$
\lim _{\lambda \rightarrow \infty} P_{G_{\lambda} w}^{1}\left\{\tau\left(1-\exp \left\{e^{a \lambda / 2}(w(1)+\varepsilon)\right\}\right)>\exp \left\{e^{a \lambda / 2} C^{\prime \prime}\right\}\right\}=1 .
$$

By (4.19) and (4.23), we get

$$
\lim _{\lambda \rightarrow \infty} P_{G_{\lambda} w}^{0}\left\{X\left(e^{\lambda(1-2 a)}\right)>1-\exp \left\{e^{a \lambda / 2}(w(1)+\varepsilon)\right\}\right\}=1 .
$$

Combining (4.21) and (4.24), we obtain, for any $\varepsilon \in(0,-w(1))$

$$
\lim _{\lambda \rightarrow \infty} P_{G_{\lambda} w}^{0}\left\{-\exp \left\{e^{a \lambda / 2}(w(1)+\varepsilon)\right\}<X\left(e^{\lambda(1-2 a)}\right)-1<\varepsilon\right\}=1 .
$$

Therefore we get (iii).

\section{Proof of Theorem 1.3}

We obtain Theorem 1.3 from the following proposition in the same way as obtaining Theorem 1.1 from Proposition 2.2. 
Proposition 5.1. (i) There exists a subset $\left(\mathbb{A}_{I}^{\prime}\right)^{\#}$ of $\mathbb{A}_{I}^{\prime}$ with $P\left\{\mathbb{A}_{I}^{\prime} \backslash\left(\mathbb{A}_{I}^{\prime}\right)^{\#}\right\}=0$ such that, for any $w \in\left(\mathbb{A}_{I}^{\prime}\right)^{\#}$ and $\varepsilon>0$

$$
\lim _{\lambda \rightarrow \infty} P_{G_{\lambda} w}^{0}\left\{e^{\lambda(H-a-\varepsilon)}<\bar{X}\left(e^{\lambda(1-2 a)}\right)<e^{\lambda(H-a+\varepsilon)} \wedge \varepsilon\right\}=1 .
$$

(ii) There exists a subset $\left(\mathbb{A}_{I I}^{\prime}\right)^{\#}$ of $\mathbb{A}_{I I}^{\prime}$ with $P\left\{\mathbb{A}_{I I}^{\prime} \backslash\left(\mathbb{A}_{I I}^{\prime}\right)^{\#}\right\}=0$ such that, for any $w \in\left(\mathbb{A}_{I I}^{\prime}\right)^{\#}, \varepsilon>0$ and $\varepsilon(\lambda)>0, \lambda>0$, satisfying $\lim _{\lambda \rightarrow \infty} \varepsilon(\lambda)=0$ and $\liminf _{\lambda \rightarrow \infty} \lambda(\log \lambda)^{-1} \varepsilon(\lambda)>2$

$$
\lim _{\lambda \rightarrow \infty} P_{G_{\lambda} w}^{0}\left\{e^{\lambda(H-a-\varepsilon)}<\bar{X}\left(e^{\lambda(1-2 a)}\right)<e^{\lambda(H-a+\varepsilon(\lambda))} \wedge \varepsilon\right\}=1 .
$$

(iii) There exists a subset $\mathbb{D}^{\#}$ of $\mathbb{D}$ with $P\left\{\mathbb{D} \backslash \mathbb{D}^{\#}\right\}=0$ such that, for any $w \in \mathbb{D}^{\#}$ and $\varepsilon>0$

$$
\lim _{\lambda \rightarrow \infty} P_{G_{\lambda} w}^{0}\left\{f_{\lambda}(\varepsilon)<\bar{X}\left(e^{\lambda(1-2 a)}\right)-1<g_{\lambda}(\varepsilon) \wedge \varepsilon\right\}=1
$$

where

$$
\begin{aligned}
& f_{\lambda}(\varepsilon)=f_{\lambda}(\varepsilon, w)=\exp \left\{-e^{a \lambda / 2}(w(1)+\varepsilon)\right\}, \\
& g_{\lambda}(\varepsilon)=g_{\lambda}(\varepsilon, w)=\exp \left\{-e^{a \lambda / 2}(w(1)-\varepsilon)\right\} .
\end{aligned}
$$

(iv) There exists a subset $\mathbb{C}^{\#}$ of $\mathbb{C}$ with $P\left\{\mathbb{C} \backslash \mathbb{C}^{\#}\right\}=0$ such that, for any $w \in \mathbb{C}^{\#}$ and $\varepsilon>0$

$$
\lim _{\lambda \rightarrow \infty} P_{G_{\lambda} w}^{0}\left\{h_{\lambda}(\varepsilon)<\bar{X}\left(e^{\lambda(1-2 a)}\right)-1<\varepsilon\right\}=1
$$

where

$$
h_{\lambda}(\varepsilon)=\exp \left\{-e^{a \lambda / 2} \varepsilon\right\}
$$

To prove Proposition 5.1, we prepare three lemmas.

LEMMA 5.2. Let $w \in \mathbb{W}$ and assume $w(1)>0$. Then for any $\varepsilon>0$ and $\xi \in(0, \varepsilon)$

$$
\lim _{\lambda \rightarrow \infty} P_{G_{\lambda} w}^{1}\left\{\tau\left(1+f_{\lambda}(\varepsilon)\right)<\exp \left\{-e^{a \lambda / 2} \xi\right\}\right\}=1 .
$$

Proof. Assume $w(1)>0$ and let $\varepsilon>0$. Then we have $f_{\lambda}(\varepsilon) \downarrow 0$ as $\lambda \rightarrow \infty$. Note that

$$
\tau\left(1+f_{\lambda}(\varepsilon) ; 1, G_{\lambda} w\right)=\tau\left(f_{\lambda}(\varepsilon) ; 0,\left(G_{\lambda} w\right)^{1}\right) .
$$

For $\eta \in(0,1)$, we set

$$
\widetilde{E}_{\lambda}=\left\{\tau\left(f_{\lambda}(\varepsilon) ; 0,\left(G_{\lambda} w\right)^{1}\right)<\tau\left(-\eta ; 0,\left(G_{\lambda} w\right)^{1}\right)\right\}
$$

and observe

$$
\widetilde{P}\left\{\widetilde{E}_{\lambda}\right\}=\frac{\eta}{\int_{1}^{1+f_{\lambda}(\varepsilon)} \exp \left\{e^{a \lambda / 2} w(x)\right\} d x+\eta} .
$$


By the definition of $f_{\lambda}(\varepsilon)$, we see that

$$
\int_{1}^{1+f_{\lambda}(\varepsilon)} \exp \left\{e^{a \lambda / 2} w(x)\right\} d x \leq \exp \left\{e^{a \lambda / 2}\left(M_{\lambda}-w(1)-\varepsilon\right)\right\},
$$

where $M_{\lambda}=\max _{1 \leq x \leq 1+f_{\lambda}(\varepsilon)} w(x)$. Since $M_{\lambda} \downarrow w(1)$ as $\lambda \rightarrow \infty$, the right-hand side of (5.6) converges to 0 as $\lambda \rightarrow \infty$. Therefore we get

$$
\lim _{\lambda \rightarrow \infty} \widetilde{P}\left\{\widetilde{E}_{\lambda}\right\}=1 .
$$

On $\widetilde{E}_{\lambda}$, by the same argument as in the proof of Lemma 3.1, the right-hand side of (5.5) is equal to

$$
\begin{aligned}
\int_{-\eta}^{f_{\lambda}(\varepsilon)} & e^{-\left(G_{\lambda} w\right)^{1}(x)} L\left(T\left(S_{\left(G_{\lambda} w\right)^{1}}\left(f_{\lambda}(\varepsilon)\right)\right), S_{\left(G_{\lambda} w\right)^{1}}(x)\right) d x \\
& \stackrel{\mathrm{d}}{=} S_{\left(G_{\lambda} w\right)^{1}}\left(f_{\lambda}(\varepsilon)\right) \int_{-\eta}^{f_{\lambda}(\varepsilon)} e^{-\left(G_{\lambda} w\right)^{1}(x)} L\left(T(1), \frac{S_{\left(G_{\lambda} w\right)^{1}}(x)}{S_{\left(G_{\lambda} w\right)^{1}}\left(f_{\lambda}(\varepsilon)\right)}\right) d x \\
\quad= & I_{\lambda}^{\prime}+I I_{\lambda}^{\prime}
\end{aligned}
$$

where

$$
\begin{aligned}
& S_{\left(G_{\lambda} w\right)^{1}}(x)=\int_{0}^{x} e^{\left(G_{\lambda} w\right)^{1}(y)} d y, \quad x \in \mathbb{R}, \\
& I_{\lambda}^{\prime}=\int_{1}^{1+f_{\lambda}(\varepsilon)} \exp \left\{e^{a \lambda / 2} w(y)\right\} d y \int_{1-\eta}^{1} L\left(T(1), \frac{S_{\left(G_{\lambda} w\right)^{1}}(z-1)}{S_{\left(G_{\lambda} w\right)^{1}}\left(f_{\lambda}(\varepsilon)\right)}\right) d z, \\
& I I_{\lambda}^{\prime}=I I I_{\lambda}^{\prime} \times I V_{\lambda}^{\prime}, \\
& I I I_{\lambda}^{\prime}=\int_{1}^{1+f_{\lambda}(\varepsilon)} \exp \left\{e^{a \lambda / 2} w(y)\right\} d y, \\
& I V_{\lambda}^{\prime}=\int_{1}^{1+f_{\lambda}(\varepsilon)} \exp \left\{-e^{a \lambda / 2} w(z)\right\} L\left(T(1), \frac{S_{\left(G_{\lambda} w\right)^{1}}(z-1)}{S_{\left(G_{\lambda} w\right)^{1}}\left(f_{\lambda}(\varepsilon)\right)}\right) d z .
\end{aligned}
$$

We note that $0<K(T(1))<\infty(\widetilde{P}$-a.s. $)$, where $K(\cdot)$ is defined in the proof of Lemma 3.1. From this, we can estimate $I_{\lambda}^{\prime}$ and $I I_{\lambda}^{\prime}$ as follows:

$$
\begin{aligned}
& I_{\lambda}^{\prime} \leq \eta K(T(1)) \exp \left\{e^{a \lambda / 2}\left(M_{\lambda}-w(1)-\varepsilon\right)\right\}, \quad \widetilde{P} \text {-a.s. }, \\
& I I_{\lambda}^{\prime} \leq K(T(1)) \exp \left\{e^{a \lambda / 2}\left\{M_{\lambda}-C_{\lambda}-2(w(1)+\varepsilon)\right\}\right\}, \quad \widetilde{P} \text {-a.s. },
\end{aligned}
$$

where $C_{\lambda}=\min _{1 \leq x \leq 1+f_{\lambda}(\varepsilon)} w(x)$. Using $M_{\lambda} \downarrow w(1)($ as $\lambda \rightarrow \infty)$ and $C_{\lambda} \uparrow w(1)$ (as $\lambda \rightarrow \infty)$, we get

$$
\begin{aligned}
& \limsup _{\lambda \rightarrow \infty} e^{-a \lambda / 2} \log I_{\lambda}^{\prime} \leq-\varepsilon, \quad \widetilde{P} \text {-a.s. } \\
& \limsup _{\lambda \rightarrow \infty} e^{-a \lambda / 2} \log I I_{\lambda}^{\prime} \leq-2(w(1)+\varepsilon)<-\varepsilon, \quad \widetilde{P} \text {-a.s. }
\end{aligned}
$$


Therefore, for any $\xi \in(0, \varepsilon)$ we have

$$
\lim _{\lambda \rightarrow \infty} \widetilde{P}\left\{I_{\lambda}^{\prime}+I I_{\lambda}^{\prime}<\exp \left\{-e^{a \lambda / 2} \xi\right\}\right\}=1 .
$$

By (5.5) and (5.7)-(5.9), we obtain

$$
\lim _{\lambda \rightarrow \infty} \widetilde{P}\left\{\tau\left(1+f_{\lambda}(\varepsilon) ; 1, G_{\lambda} w\right)<\exp \left\{-e^{a \lambda / 2} \xi\right\}\right\}=1,
$$

which is equivalent to (5.4).

Lemma 5.3. Let $w \in \mathbb{W}$ and assume $w(1)>0$. Then for any $\varepsilon \in(0, w(1))$ and $p<0$

$$
\lim _{\lambda \rightarrow \infty} P_{G_{\lambda} w}^{0}\left\{\tau\left(1+g_{\lambda}(\varepsilon)\right)>\tau\left(\lambda^{2} e^{-a \lambda} p\right)\right\}=1
$$

Proof. Assume $w(1)>0$ and let $\varepsilon \in(0, w(1))$. Then we have $g_{\lambda}(\varepsilon) \downarrow 0$ as $\lambda \rightarrow \infty$. For any $p<0$ we observe

$$
\begin{aligned}
P_{G_{\lambda} w}^{0} & \left\{\tau\left(1+g_{\lambda}(\varepsilon)\right)>\tau\left(\lambda^{2} e^{-a \lambda} p\right)\right\} \\
& =\frac{1+\int_{1}^{1+g_{\lambda}(\varepsilon)} \exp \left\{e^{a \lambda / 2} w(x)\right\} d x}{\lambda^{2} e^{-a \lambda} \int_{p}^{0} e^{\lambda w(x)} d x+1+\int_{1}^{1+g_{\lambda}(\varepsilon)} \exp \left\{e^{a \lambda / 2} w(x)\right\} d x} .
\end{aligned}
$$

Setting $C_{\lambda}^{\prime}=\min _{1 \leq x \leq 1+g_{\lambda}(\varepsilon)} w(x)$, we have

$$
\int_{1}^{1+g_{\lambda}(\varepsilon)} \exp \left\{e^{a \lambda / 2} w(x)\right\} d x \geq \exp \left\{e^{a \lambda / 2}\left(C_{\lambda}^{\prime}-w(1)+\varepsilon\right)\right\} .
$$

Since $C_{\lambda}^{\prime} \uparrow w(1)$ as $\lambda \rightarrow \infty$, we see that the right-hand side of (5.11) tends to $\infty$ as $\lambda \rightarrow \infty$ and we obtain (5.10).

The following lemma can be shown by the same argument as in the proof of Lemma 5.2.

LEMmA 5.4. Let $w \in \mathbb{W}$ and assume $w(1)<0$, and let $\varepsilon>0$ and $J=\max \{w(1)-$ $\varepsilon,-2 \varepsilon\}$. Then for any $\xi \in(0,-J)$

$$
\lim _{\lambda \rightarrow \infty} P_{G_{\lambda} w}^{1}\left\{\tau\left(1+h_{\lambda}(\varepsilon)\right)<\exp \left\{-e^{a \lambda / 2} \xi\right\}\right\}=1 .
$$

Let us now prove Proposition 5.1.

Proof OF Proposition 5.1. First we show (i) by employing the method in [3, Lemma 6.1]. Let $w \in \mathbb{A}^{\prime}$. We observe, for any sufficiently small $\varepsilon>0$

$$
\begin{aligned}
P_{G_{\lambda} w}^{0}\left\{\tau\left(e^{\lambda(H-a-\varepsilon)}\right)<\tau\left(\lambda^{2} e^{-a \lambda} \zeta_{1-\varepsilon / 2}\right)\right\} & =\frac{\lambda^{2} \int_{\zeta_{1-\varepsilon / 2}}^{0} e^{\lambda w(x)} d x}{e^{\lambda(H-\varepsilon)}+\lambda^{2} \int_{\zeta_{1-\varepsilon / 2}}^{0} e^{\lambda w(x)} d x} \\
& \rightarrow 1, \quad \text { as } \lambda \rightarrow \infty,
\end{aligned}
$$


since $\lim _{\lambda \rightarrow \infty} \lambda^{-1} \log \int_{\zeta_{1-\varepsilon / 2}}^{0} e^{\lambda w(x)} d x=\max _{\zeta_{1-\varepsilon / 2} \leq x \leq 0} w(x) \geq H-\varepsilon / 2>H-\varepsilon$. Combining this with Lemma 4.1, we have

$$
\lim _{\lambda \rightarrow \infty} P_{G_{\lambda} w}^{0}\left\{e^{\lambda(H-a-\varepsilon)}<\bar{X}\left(e^{\lambda(1-2 a)}\right)\right\}=1 .
$$

Moreover, in the case $w \in \mathbb{A}_{I}^{\prime}$ we have, for any $\varepsilon \in(0, a-H)$ satisfying $\min _{\zeta_{1+\varepsilon / 2} \leq x \leq \zeta} w(x)>V$

$$
\lim _{\lambda \rightarrow \infty} P_{G_{\lambda} w}^{0}\left\{\tau\left(e^{\lambda(H-a+\varepsilon)}\right)>\tau\left(\lambda^{2} e^{-a \lambda} \zeta_{1+\varepsilon / 2}\right)\right\}=1,
$$

since $\max _{\zeta_{1+\varepsilon / 2} \leq x \leq 0} w(x)=H+\varepsilon / 2<H+\varepsilon$. Combining this with Lemma 4.2, we have, for any $\varepsilon \in(0, a-H)$

$$
\lim _{\lambda \rightarrow \infty} P_{G_{\lambda} w}^{0}\left\{\bar{X}\left(e^{\lambda(1-2 a)}\right)<e^{\lambda(H-a+\varepsilon)}\right\}=1 .
$$

By (5.12) and (5.13), we obtain (i).

In the case $w \in \mathbb{A}_{I I}^{\prime}$ we show (ii) by improving the method in [3, Lemma 6.1]. In this case we have, for any sufficiently small $\varepsilon>0 \max _{\zeta_{1+\varepsilon} \leq x \leq 0} w(x)=H$ and therefore for all sufficiently large $\lambda>0$ satisfying $\varepsilon(\lambda)<a-H$

$$
\begin{aligned}
P_{G_{\lambda} w}^{0}\left\{\tau\left(e^{\lambda(H-a+\varepsilon(\lambda))}\right)>\tau\left(\lambda^{2} e^{-a \lambda} \zeta_{1+\varepsilon}\right)\right\} & =\frac{e^{\lambda(H+\varepsilon(\lambda))}}{\lambda^{2} \int_{\zeta_{1+\varepsilon}}^{0} e^{\lambda w(x)} d x+e^{\lambda(H+\varepsilon(\lambda))}} \\
& \geq \frac{e^{\lambda(H+\varepsilon(\lambda))}}{\lambda^{2}\left|\zeta_{1+\varepsilon}\right| e^{\lambda H}+e^{\lambda(H+\varepsilon(\lambda))}} \\
& =\frac{1}{\lambda^{2}\left|\zeta_{1+\varepsilon}\right| e^{-\lambda \varepsilon(\lambda)}+1} .
\end{aligned}
$$

We notice that there exists $\xi_{0}>0$ such that for all sufficiently large $\lambda>0 \varepsilon(\lambda)>(2+$ $\left.\xi_{0}\right) \lambda^{-1} \log \lambda$. Therefore the right-hand side of (5.14) converges to 1 as $\lambda \rightarrow \infty$. Combining this with Lemma 4.2, we get

$$
\lim _{\lambda \rightarrow \infty} P_{G_{\lambda} w}^{0}\left\{\bar{X}\left(e^{\lambda(1-2 a)}\right)<e^{\lambda(H-a+\varepsilon(\lambda))}\right\}=1 .
$$

By (5.12) and (5.15), we obtain (ii).

Next we prove (iii). Let $w \in \mathbb{D}$ and $\varepsilon>0$. In this case, in Lemma 3.4 we notice $0 \leq J_{I I}<1-2 a$. Therefore, by combining Lemma 3.4 with Lemma 5.2, we get, for any sufficiently small $\delta>0$

$$
\lim _{\lambda \rightarrow \infty} P_{G_{\lambda} w}^{0}\left\{\tau\left(1+f_{\lambda}(\varepsilon)\right)<e^{\lambda(1-2 a-\delta)}\right\}=1 .
$$

On the other hand, we let $\varepsilon \in(0, w(1))$. By combining Lemma 5.3 with Lemma 4.2 in the case $w \in \mathbb{A}^{\prime \prime} \cap\left(\mathbb{A}^{\prime}\right)^{c}$ and with (4.16) in the case $w \in \mathbb{B}$, we have, for any sufficiently small 
$\delta^{\prime}>0$

$$
\lim _{\lambda \rightarrow \infty} P_{G_{\lambda} w}^{0}\left\{\tau\left(1+g_{\lambda}(\varepsilon)\right)>e^{\lambda\left(1-2 a+\delta^{\prime}\right)}\right\}=1 .
$$

By (5.16) and (5.17), we obtain, for any $\varepsilon \in(0, w(1))$

$$
\lim _{\lambda \rightarrow \infty} P_{G_{\lambda} w}^{0}\left\{f_{\lambda}(\varepsilon)<\bar{X}\left(e^{\lambda(1-2 a)}\right)-1<g_{\lambda}(\varepsilon)\right\}=1 .
$$

Therefore we obtain (iii).

As to (iv), we get (5.3) by using (4.19), Lemma 5.4 and (4.20).

\section{Proof of Theorem 1.4}

Theorem 1.4 is obtained from the following proposition.

Proposition 6.1. (i) There exists a subset $\mathbb{A}^{\#}$ of $\mathbb{A}$ with $P\left\{\mathbb{A} \backslash \mathbb{A}^{\#}\right\}=0$ such that, for any $w \in \mathbb{A}^{\#}, \varepsilon>0$ and $\varepsilon(\lambda)>0, \lambda>0$, satisfying $\lim _{\lambda \rightarrow \infty} \varepsilon(\lambda)=0$ and $\liminf _{\lambda \rightarrow \infty} \lambda(\log \lambda)^{-1} \varepsilon(\lambda)>4$

$$
\lim _{\lambda \rightarrow \infty} P_{G_{\lambda} w}^{0}\left\{\lambda^{2} e^{-a \lambda} \zeta_{1+\varepsilon}<\underline{X}\left(e^{\lambda(1-2 a)}\right)<\lambda^{2} e^{-a \lambda} \zeta_{1-\varepsilon(\lambda)}\right\}=1 .
$$

(ii) There exists a subset $\mathbb{B}^{\#}$ of $\mathbb{B}$ with $P\left\{\mathbb{B} \backslash \mathbb{B}^{\#}\right\}=0$ such that, for any $w \in \mathbb{B}^{\#}, \varepsilon>0$ and $\varepsilon(\lambda)>0, \lambda>0$, satisfying $\lim _{\lambda \rightarrow \infty} \varepsilon(\lambda)=0$ and $\liminf _{\lambda \rightarrow \infty} \lambda(\log \lambda)^{-1} \varepsilon(\lambda)>2$

$$
\lim _{\lambda \rightarrow \infty} P_{G_{\lambda} w}^{0}\left\{\lambda^{2} e^{-a \lambda} \sigma(1-a+\varepsilon)<\underline{X}\left(e^{\lambda(1-2 a)}\right)<\lambda^{2} e^{-a \lambda} \sigma(1-a-\varepsilon(\lambda))\right\}=1 .
$$

(iii) There exists a subset $\mathbb{C}^{\#}$ of $\mathbb{C}$ with $P\left\{\mathbb{C} \backslash \mathbb{C}^{\#}\right\}=0$ such that, for any $w \in \mathbb{C}^{\#}$ and $\varepsilon(\lambda)>0, \lambda>0$, satisfying $\lim _{\lambda \rightarrow \infty} \varepsilon(\lambda)=0$ and $\liminf _{\lambda \rightarrow \infty} \lambda(\log \lambda)^{-1} \varepsilon(\lambda)>2$

$$
\lim _{\lambda \rightarrow \infty} P_{G_{\lambda} w}^{0}\left\{\lambda^{2} e^{-a \lambda} \sigma(a)<\underline{X}\left(e^{\lambda(1-2 a)}\right)<\lambda^{2} e^{-a \lambda} \sigma(a-\varepsilon(\lambda))\right\}=1 .
$$

Proof. First we prove (i) by improving Lemma 4.1. Let $w \in \mathbb{A}$. In the case $M<$ $V+1$, we can apply Lemma 3.1 to $p=\zeta, p_{\lambda}=\zeta_{1-\varepsilon(\lambda)}$ and $x_{0}=0$ because of (1.6) or the definition of $\mathbb{A}^{\prime \prime}$. In this case the assertion (3.1) holds for

$$
J(\lambda)=1-2 a-\varepsilon(\lambda)+4 \lambda^{-1} \log \lambda+\lambda^{-1} \log \log \lambda,
$$

since $V_{\lambda}$ in Lemma 3.1 is equal to $V$ for all sufficiently large $\lambda>0$ and (1.1) holds. We notice that there exists $\xi_{1}>0$ such that for all sufficiently large $\lambda>0$

$$
\varepsilon(\lambda)>\left(4+\xi_{1}\right) \lambda^{-1} \log \lambda .
$$

By (6.4) and (6.5), the assertion (3.1) yields

$$
\lim _{\lambda \rightarrow \infty} P_{G_{\lambda} w}^{0}\left\{\tau\left(\lambda^{2} e^{-a \lambda} \zeta_{1-\varepsilon(\lambda)}\right)<e^{\lambda\left(1-2 a-\xi \lambda^{-1} \log \lambda\right)}\right\}=1
$$


for any $\xi \in\left(0, \xi_{1}\right)$. By (6.6) and Lemma 4.2, we obtain (6.1).

In the case $M \geq V+1$, we can apply Lemma 3.1 to $p=\zeta, p_{\lambda}=\zeta_{1-\varepsilon(\lambda)}$ and $x_{0}=c_{n-1}$ defined in the proof of Lemma 4.1. By the same argument as above, we get, for any $\xi \in\left(0, \xi_{1}\right)$

$$
\lim _{\lambda \rightarrow \infty} P_{G_{\lambda} w}^{\lambda^{2} e^{-a \lambda} c_{n-1}}\left\{\tau\left(\lambda^{2} e^{-a \lambda} \zeta_{1-\varepsilon(\lambda)}\right)<e^{\lambda\left(1-2 a-\xi \lambda^{-1} \log \lambda\right)}\right\}=1 .
$$

By (4.5), (6.7) and the strong Markov property, we obtain (6.6) for any $\xi \in\left(0, \xi_{1}\right)$ and therefore (6.1) in this case, too.

Next we prove (ii). Let $w \in \mathbb{B}$. We can apply Lemma 3.1 to $p=\sigma(1-a), p_{\lambda}=$ $\sigma(1-a-\varepsilon(\lambda))$ and $x_{0}=0$. In this case, in the lemma, for all sufficiently large $\lambda>0$ $V_{\lambda}=\min _{\sigma(1-a) \leq x \leq 0} w(x)>-a$. Therefore the assertion (3.1) holds for

$$
J(\lambda)=1-2 a-\varepsilon(\lambda)+2 \lambda^{-1} \log \lambda+\lambda^{-1} \log \log \lambda .
$$

We notice that there exists $\xi_{2}>0$ such that for all sufficiently large $\lambda>0$

$$
\varepsilon(\lambda)>\left(2+\xi_{2}\right) \lambda^{-1} \log \lambda .
$$

By (6.8) and (6.9), the assertion (3.1) yields

$$
\lim _{\lambda \rightarrow \infty} P_{G_{\lambda} w}^{0}\left\{\tau\left(\lambda^{2} e^{-a \lambda} \sigma(1-a-\varepsilon(\lambda))\right)<e^{\lambda\left(1-2 a-\xi \lambda^{-1} \log \lambda\right)}\right\}=1
$$

for any $\xi \in\left(0, \xi_{2}\right)$. From this and (4.16), we get (6.2).

Finally we prove (iii). Let $w \in \mathbb{C}$. By (3.14), (4.19) and (4.23), we have, for some $C^{\prime \prime}>0$

$$
\lim _{\lambda \rightarrow \infty} P_{G_{\lambda} w}^{0}\left\{\tau\left(\lambda^{2} e^{-a \lambda} \sigma(a)\right)>\exp \left\{e^{a \lambda / 2} C^{\prime \prime}\right\}\right\}=1 .
$$

On the other hand, we set $q_{\lambda}=\sigma(a-\varepsilon(\lambda))$. Then we observe

$$
P_{G_{\lambda} w}^{0}\left\{\tau\left(\lambda^{2} e^{-a \lambda} q_{\lambda}\right)<\tau(1)\right\}=\frac{e^{a \lambda}}{e^{a \lambda}+\lambda^{2} \int_{q_{\lambda}}^{0} e^{\lambda w(x)} d x}
$$

and

$$
\begin{aligned}
e^{-a \lambda} \lambda^{2} \int_{q_{\lambda}}^{0} e^{\lambda w(x)} d x & \leq\left|q_{\lambda}\right| \exp \left\{\lambda\left(w\left(q_{\lambda}\right)-a+2 \lambda^{-1} \log \lambda\right)\right\} \\
& \leq|\sigma(a)| \exp \left\{\lambda\left(-\varepsilon(\lambda)+2 \lambda^{-1} \log \lambda\right)\right\}
\end{aligned}
$$

The right-hand side of (6.11) converges to 0 as $\lambda \rightarrow \infty$, since (6.9) holds in this case, too. As a result, we have

$$
\lim _{\lambda \rightarrow \infty} P_{G_{\lambda} w}^{0}\left\{\tau\left(\lambda^{2} e^{-a \lambda} q_{\lambda}\right)<\tau(1)\right\}=1 .
$$

By (4.19), (6.12) and (6.10), we get (6.3). 
ACKNowledgment. The author has considered the present model by being motivated by the conversation with Professor N. Konno, to whom she expresses her gratitude. She also thanks Professor Y. Tamura for his encouragement.

\section{References}

[ 1 ] Brox, T., A one-dimensional diffusion process in a Wiener medium, Ann. Probab. 14 (1986), 1206-1218.

[ 2 ] Itô, K. and McKean, H. P., Diffusion Processes and their Sample Paths, Springer-Verlag, 1965.

[ 3 ] KAWAZU, K. and SuzUKi, Y., Limit theorems for a diffusion process with a one-sided Brownian potential, Journal of Applied Probability $\mathbf{4 3}$ (2006), 997-1012.

[ 4 ] KawaZU, K., SuzUki, Y. and TAnaka, H., A diffusion process with a one-sided Brownian potential, Tokyo J. Math. 24 (2001), 211-229.

[ 5 ] KawazU, K., Tamura, Y. and Tanaka, H., One-dimensional diffusions and random walks in random environments, In Probability Theory and Mathematical Statistics, eds. S. Watanabe and Yu. V. Prokhorov, Lecture Notes in Math. 1299 (1988), Springer, 170-184.

[6] KawazU, K., Tamura, Y. and Tanaka, H., Limit theorems for one-dimensional diffusions and random walks in random environments, Probab. Theory Related Fields 80 (1989), 501-541.

[ 7 ] Schumacher, S., Diffusions with random coefficients. In Particle Systems, Random Media and Large Deviations, ed. R. Durrett, Contemp. Math. 41 (1985), American Math. Soc., 351-356.

[ 8 ] SUZUKI, Y., A diffusion process with a random potential consisting of two self-similar processes with different indices, Tokyo J. Math. 31 (2008), 511-532.

Present Address:

KeIo University SCHOol of Medicine,

HIYOSHI, KOUHOKU-KU, YOKOHAMA 223-8521, JAPAN. 